\title{
Adult neurogenesis mediates forgetting of multiple types of memory in the rat
}

\author{
Gavin A. Scott ${ }^{1}$, Dylan J. Terstege ${ }^{1}$, Andrew J. Roebuck ${ }^{2}$, Kelsea A. Gorzo ${ }^{1}$, Alex P. Vu ${ }^{1}$, John G. Howland ${ }^{3}$ and \\ Jonathan R. Epp ${ }^{1 *}$
}

\begin{abstract}
The formation and retention of hippocampus-dependent memories is impacted by neurogenesis, a process that involves the production of new neurons in the dentate gyrus of the hippocampus. Recent studies demonstrate that increasing neurogenesis after memory formation induces forgetting of previously acquired memories. Neurogenesisinduced forgetting was originally demonstrated in mice, but a recent report suggests that the same effect may be absent in rats. Although a general species difference is possible, other potential explanations for these incongruent findings are that memories which are more strongly reinforced become resilient to forgetting or that perhaps only certain types of memories are affected. Here, we investigated whether neurogenesis-induced forgetting occurs in rats using several hippocampus-dependent tasks including contextual fear conditioning (CFC), the Morris Water Task (MWT), and touchscreen paired associates learning (PAL). Neurogenesis was increased following training using voluntary exercise for 4 weeks before recall of the previous memory was assessed. We show that voluntary running causes forgetting of context fear memories in a neurogenesis-dependent manner, and that neurogenesis-induced forgetting is present in rats across behavioral tasks despite differences in complexity or reliance on spatial, context, or object memories. In addition, we asked whether stronger memories are less susceptible to forgetting by varying the strength of training. Even with a very strong training protocol in the CFC task, we still observed enhanced forgetting related to increased neurogenesis. These results suggest that forgetting due to neurogenesis is a conserved mechanism that aids in the clearance of memories.
\end{abstract}

Keywords: Neurogenesis, Forgetting, Hippocampus, Morris Water Task, Contextual fear conditioning, Paired Associates Learning, Touchscreens

\section{Introduction}

Hippocampal neurogenesis plays a critical role in longterm memory, but its precise roles are not fully understood. Ablation of adult neurogenesis, or impairing the synaptic integration of adult-born neurons, impairs the acquisition of new memories [1-3] and enhancement of neurogenesis improves subsequent learning [4]. Only

\footnotetext{
*Correspondence: jonathan.epp1@ucalgary.ca

${ }^{1}$ Department of Cell Biology and Anatomy, Hotchkiss Brain Institute, Cumming School of Medicine, HMRB 162, Health Sciences Centre, University of Calgary, 3330 Hospital Drive NW, Calgary, ABT2N 4N1, Canada

Full list of author information is available at the end of the article
}

recently has significant attention been paid to the retrograde effects of adult neurogenesis on existing memories. Recent evidence has demonstrated that adult neurogenesis mediates forgetting of existing memories. We define "forgetting", for present purposes, as simply the inability to recall information that was once recalled previously [5]. First predicted computationally [6], several experimental studies have confirmed that increased neurogenesis causes retrograde degradation of long-term memory in different hippocampus (HPC)-dependent tasks, across rodent species, and across the lifespan [7-11]. A beneficial outcome of this neurogenesis-induced reduction in memory retention 
is that it allows for the encoding of new memories free from proactive interference caused by older memories [8]. The presence of this phenomenon in three different rodent species (mouse, degu, guinea pig) suggests that it is a conserved mechanism for ameliorating proactive memory interference.

Contrary to computational models [6] and experiments using mice $[7,8,10]$, a recent study using rats found that increasing adult neurogenesis did not cause forgetting of a spatial memory in the Morris Water Task (MWT) [12]. These authors trained rats in the MWT and increased neurogenesis using voluntary wheel running. Despite exercise causing $\mathrm{a} \sim$ twofold increase in the number of new neurons in the dentate gyrus (DG), there was no difference in retention of the original platform location, indicating that increasing neurogenesis did not impair retention of a previously acquired spatial memory. On this basis, the authors called into question whether neurogenesis-induced forgetting is present in rats and thus, whether the phenomenon is conserved across species.

To date, neurogenesis-induced forgetting in the rat has not been assessed in any other behavioral tasks. However, neurogenesis-induced forgetting in mice has been demonstrated in the MWT $[7,8]$, contextual fear conditioning (CFC) [7, 9], the Barnes maze [7, 11], inhibitory avoidance [9], and odor-context paired associates learning [8]. Thus, it is premature to conclude that neurogenesis-induced forgetting is absent in rats on the basis of a single behavioral assay.

Additionally, there were major differences between the MWT training protocols used by Kodali et al. [12] and Epp et al. [8]. Kodali et al. [12] conducted a greater number of days of MWT training and used longer trials. Overtraining of the MWT is known to increase mossy fiber plasticity [13] and increase complexity of CA3 dendrites [14]. This could possibly lead to a more robust circuit supporting the memory. Stronger training protocols can make memory more resistant to neurogenesisinduced forgetting [7] and even to HPC lesions [15-17]. Hence, the null findings in rats [12] could be a result of overtraining and a viable comparison would require the use of an equivalent training protocol to that used in the mouse studies $[7,8]$.

Here, we investigated the existence of neurogenesisinduced forgetting in the rat. Using voluntary exercise as a reliable means of increasing neurogenesis, we assessed multiple HPC-dependent behavioral tasks and also investigated different strengths of memory training. We provide robust evidence that neurogenesis-induced forgetting is present in the rat. Thus, this phenomenon is not unique to mice and may be an evolutionarily conserved mechanism for managing memory interference in the HPC.

\section{Materials and methods}

\section{Subjects}

A total of 152 male adult Long Evans rats (Charles River Laboratories, Kingston, NY, USA) were used in the experiments. Ninety-six were used in the CFC experiments, 28 were used in the MWT experiment, and 28 were used in paired associates learning (PAL). Rats were pair-housed in standard cages on a 12:12-h lighting cycle and had ad libitum access to standard rat chow and water except in the case of rats used in the PAL experiment, which underwent food restriction to reduce their body weight to $90 \%$ of their free feeding weight. All experiments and procedures were approved by institutional Animal Care Committees and conformed to institutional and national ethical standards.

\section{Manipulation of neurogenesis}

Voluntary wheel running was used as a manipulation because it causes a reliable increase in neurogenesis [4, $7,8,18,19]$ and although running has other effects on the brain, the forgetting effect has been shown to occur specifically due to increases in neurogenesis [8]. Half of the rats in each experiment were provided with continuous access to running wheels (Scurry Rat Activity Wheel, Lafayette Instruments, Lafayette, IN, USA) in specialized cages in which rats were pair-housed. The amount of running was monitored in a subset of cages with odometers. Sedentary control rats remained pair-housed in standard caging.

To determine whether any changes in memory were related to neurogenesis or some other effect of running, we administered temozolomide (TMZ; Biosynth Carbosynth, San Diego, CA, USA) to reduce neurogenesis. Rats were injected with $25 \mathrm{mg} / \mathrm{kg}$ TMZ or vehicle (10\% DMSO in $0.9 \%$ Saline). Injections were given on 3 consecutive days per week followed by 4 days with no injection. Half of the rats from each treatment condition were sedentary while the other half were given running wheels. Using this design, the administration of TMZ in the runners is expected to block the increases in neurogenesis [11].

\section{Contextual fear conditioning}

Fear conditioning was conducted in sound attenuated chambers with shocks delivered from a shock generator to a grated floor and freezing monitored via proprietary software (Med Associates Inc., Fairfax, VT, USA). Rats were trained with two different protocols: a weak training protocol or a strong training protocol. Weak training (Figs. 1A, 2A) involved the delivery of 3 shocks $(1 \mathrm{~mA}$, $2 \mathrm{~s}$ ) during a single $5 \mathrm{~min}$ session. Rats were given $2 \mathrm{~min}$ to explore the chamber prior to the delivery of the first shock with subsequent shocks spaced 1 min apart. Following the third shock, the rats remained in the chamber 


\begin{abstract}
(See figure on next page.)
Fig. 1 A Schematic representation of the behavior apparatus and experimental timeline used in contextual fear conditioning (CFC). B Representative photomicrographs of DCX + cells (green) in the DG (DAPl; blue) of rats in the sedentary + vehicle (SED + VEH), runner + vehicle $(R U N+V E H)$, sedentary $+T M Z$ (SED + TMZ), and runner + TMZ (RUN + TMZ) groups. C DCX + cells $/ \mathrm{mm}^{2}$ after sedentary control or running and vehicle or TMZ treatment. Upper graph shows individual data points in swarm plots with means ( \pm SD; gapped vertical line). Lower graph shows effect size (Cohen's d) as a black circle with vertical lines and gaussian distributions for the bootstrap 95\% Cl. Neurogenesis was substantially increased by running, and conversely, was decreased by TMZ. Moreover, this effect of TMZ was most striking in the running groups, with the RUN + TMZ group exhibiting unchanged neurogenesis relative to the SED +TMZ group, but reduced neurogenesis relative to the RUN + VEH group. D \% Freezing in CFC training prior to manipulation of neurogenesis. Upper graph shows individual data points in swarm plots with means ( \pm SD; gapped vertical line). Lower graph shows effect size (Cohen's d) as a black circle with vertical lines and gaussian distributions for the bootstrap $95 \%$ Cl. All 4 groups exhibited equivalent levels of freezing during conditioning. E \% Freezing during CFC testing after manipulation of neurogenesis. Upper graph shows individual data points in swarm plots with means ( \pm SD; gapped vertical line). Lower graph shows effect size (Cohen's d) as a black circle with vertical lines and gaussian distributions for the bootstrap $95 \% \mathrm{Cl}$. Freezing was significantly reduced in the RUN $+\mathrm{VEH}$ group relative to the SED + VEH group with no other significant differences being present. The results show that running causes forgetting relative to sedentary control, and that this effect is blocked by $\mathrm{TMZ}$, indicating that the forgetting effect is dependent on increases in neurogenesis
\end{abstract}

for $1 \mathrm{~min}$ before being removed to their homecage. Freezing was measured over the entire duration of the conditioning session. The strong training protocol (Fig. 2B) involved the repetition of the previously described weak training protocol over 3 consecutive days resulting in a total of 9 shocks. Following manipulations of neurogenesis, rats were returned to the fear conditioning chambers for retention testing in which freezing was monitored over a 5 min session and no shocks were delivered.

\section{Morris water task}

MWT training (Fig. 3A) was conducted in a pool $(180 \mathrm{~cm}$ in diameter) filled with $21{ }^{\circ} \mathrm{C}$ water and made opaque using non-toxic white tempera paint. A Plexiglas escape platform, $10 \mathrm{~cm}$ in diameter, was submerged $2 \mathrm{~cm}$ below the surface of the water. Behavior was monitored using an overhead camera and Any-Maze tracking software (Stoelting Co., Wood Lane, IL). Initial training consisted of 5 daily sessions of 4 trials per day. During initial training, rats were placed in one of the non-platform quadrants of the pool and allowed to swim until they located the platform, which was always located in the NW quadrant, or for a maximum of $60 \mathrm{~s}$ after which rats were placed on the platform for $15 \mathrm{~s}$. During training, we measured rats' escape latency (s) and swim distance $(\mathrm{cm})$ to reach the platform. Twenty-four hours after the final training session and prior to voluntary exercise, rats were administered a probe trial in which they were placed in the SW quadrant of the pool with the platform removed and were allowed to swim for $60 \mathrm{~s}$ to assess spatial preferences for the platform location including the percentage of time spent within the platform zone (Defined as $20 \mathrm{~cm}$ diameter circular zone surrounding the platform location), and the time (s) to the first platform crossing (swimming across the $10 \mathrm{~cm}$ diameter area originally containing the platform). Given a pool diameter of $180 \mathrm{~cm}$ and a platform zone diameter of $20 \mathrm{~cm}$, chance performance was $12 \%$ time in the platform zone, and above-chance performance was verified through the use of one-sample t-tests.

Following neurogenesis manipulation, rats' spatial memory was tested by returning them to the pool for a probe trial in which the platform was removed. Time spent in the platform quadrant was taken as a measure of memory for the original platform location. In addition, we quantified the latency to rats' first crossing of the platform in order to rule out the possibility that runners might actually remember the original platform location and reach it quickly, but then update their search strategy faster than sedentary controls due to neurogenesisinduced enhancements in cognitive flexibility.

\section{Paired associates learning Touchscreen apparatus}

PAL procedures were conducted within eight touchscreen-equipped operant conditioning chambers (Lafayette Instruments, Lafayette, IN, USA; An illustration of the interior of a chamber is shown in Fig. 4A). Each chamber was contained within a sound-attenuating box and a fan for background noise and air circulation. A live video feed of animal activity was acquired through a camera mounted within the box above the operant chamber. An interchangeable mask with 3 rectangular windows of equal size was placed flush against the touchscreen and a spring-loaded shelf was located just below these windows, requiring animals to stand when making a response.

\section{Touchscreen habituation and pretraining}

Animals were handled for at least 5 days before touchscreen habituation began. The first day of habituation involved acclimatizing the animals to the touchscreen room. Animals were transferred from the housing room to the touchscreen room and were given 5 reward pellets 


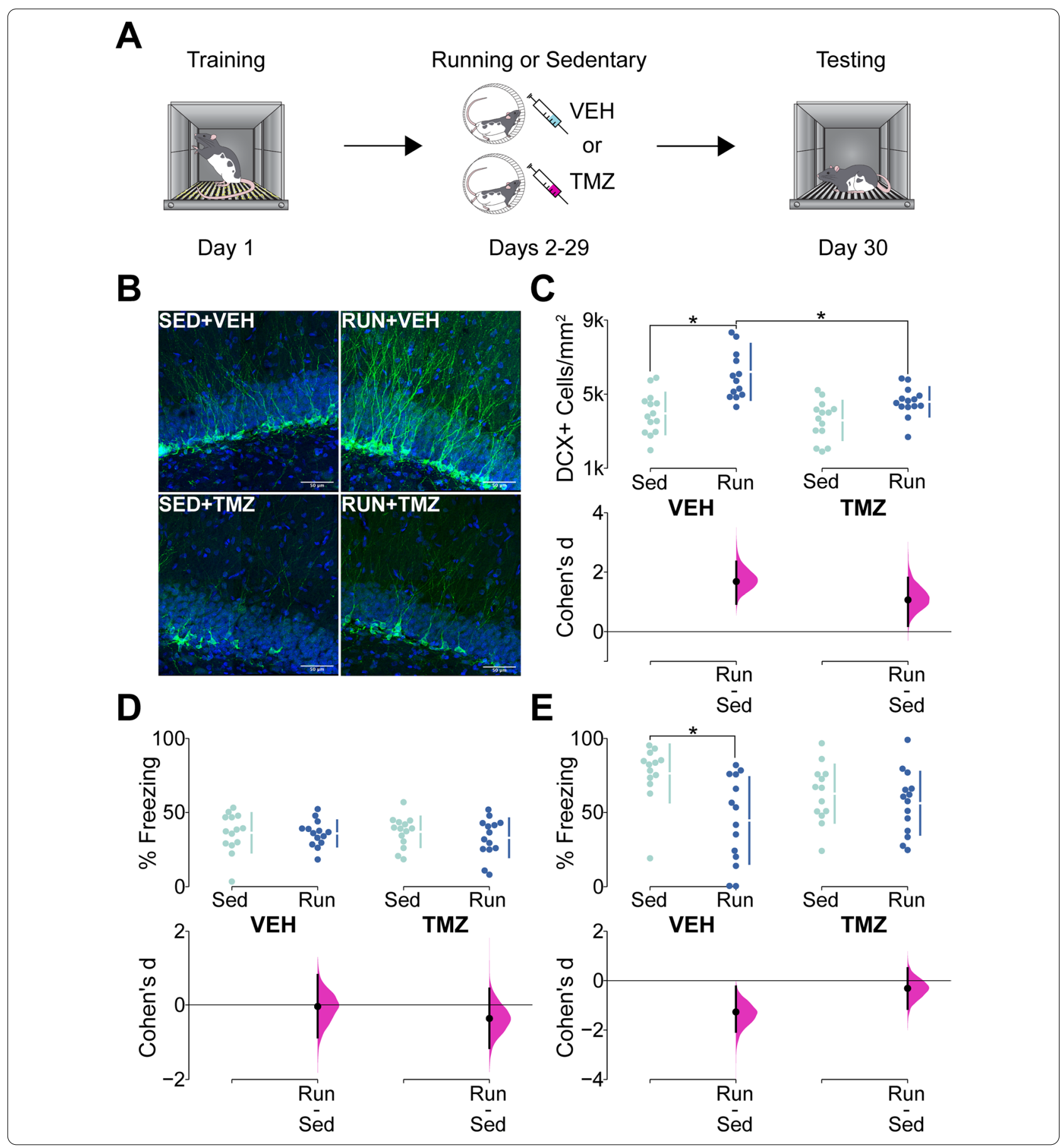

(Dustless Precision Pellets, 45 mg, Rodent Purified Diet; BioServ, NJ, USA) before being left undisturbed for $1 \mathrm{~h}$. During this period, all equipment was on and the lights were dimmed. For two additional days, animals were placed in the touchscreen chambers for $30 \mathrm{~min}$ and given 5 reward pellets. On all subsequent days of training or testing, animals were acclimatized in the touchscreen room for 15-20 min before going into the chambers.

Pretraining consisted of 4 progressive phases for training the animals to interact with the touchscreen display and receive rewards. 91) Initial Touch: One of the response windows was illuminated pseudorandomly for $30 \mathrm{~s}$ and 3 reward pellets were delivered if the rat 


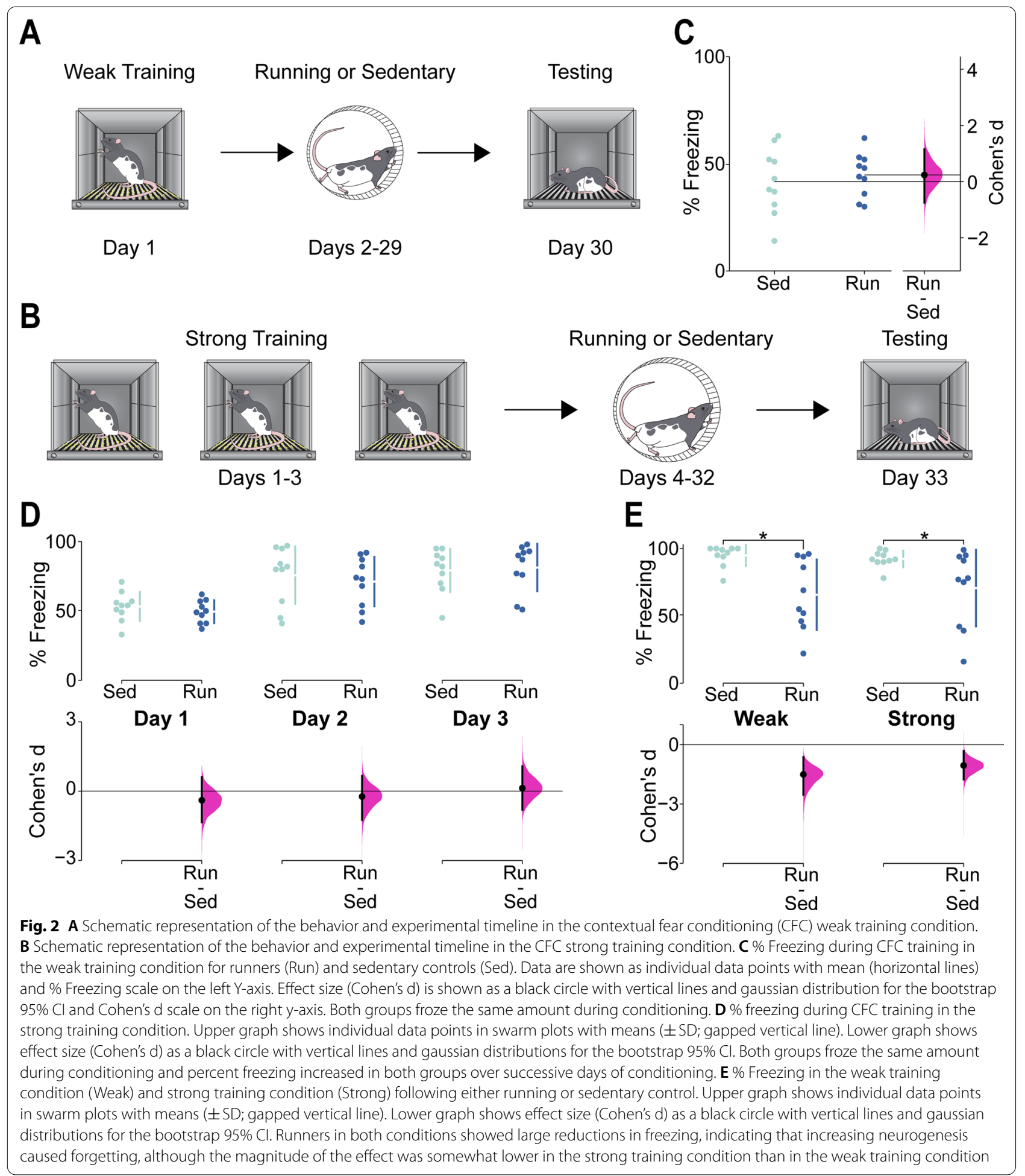

correctly touched the illuminated window during this period or only 1 reward pellet if the illuminated window was not touched. Trials were interspersed with a $20 \mathrm{~s}$ intertrial period. Criterion for Initial Touch was completion of 100 trials in $1 \mathrm{~h}$. (2) Must Touch: Identical to Initial Touch, except the animals received only 1 reward pellet and only after correct responses. The criterion for Must Touch training was 100 trials in $1 \mathrm{~h}$. (3) 


\begin{abstract}
(See figure on next page.)
Fig. 3 A Schematic representation of behavior in the MWT and the timeline of behavior training and elevation of neurogenesis. B Mean ( \pm SEM) Escape Latency during MWT training. Both groups showed equivalent reductions in latency as training progressed. C Mean ( \pm SEM) Distance Travelled to the platform during MWT training. Both groups showed equivalent decreases in distance travelled as training progressed. D \% Time in the platform zone (\% Time in Target) during pre-running (Pre) and post-running (Post) probe trials for runners (Run) and sedentary controls (Sed). Upper graph shows individual data points in swarm plots with means ( \pm SD; gapped vertical line). Chance performance (12\%) is shown with a dashed line. Lower graph shows effect size (Cohen's d) as a black circle with vertical lines and gaussian distributions for the bootstrap $95 \%$ $\mathrm{Cl}$. Prior to manipulation of neurogenesis, runners and sedentary controls spent an equivalent percentage of time in the platform zone, but after increasing neurogenesis in the running condition, runners spent significantly less time in the platform zone relative to sedentary controls, indicating that running caused forgetting of the platform location. E Latency to the first platform zone crossing (Time to Platform) during pre-running and post-running probe trials. Upper graph shows individual data points in swarm plots with means ( \pm SD; gapped vertical line). Lower graph shows effect size (Cohen's d) as a black circle with vertical lines and gaussian distributions for the bootstrap 95\% Cl. After manipulation of neurogenesis, runners took significantly longer to first cross the platform zone than controls, demonstrating that the diminished memory in runners was not an artifact of an altered search strategy
\end{abstract}

Must Initiate: Identical to Must Touch except that the animal was required to initiate each trial by nose poking in the reward port. Criterion for the Must Initiate phase was 100 trials in $1 \mathrm{~h}$. (4) Punish Incorrect: Identical to Must Initiate except that incorrect touches (i.e. touches to one of the non-illuminated windows) were punished with a $5 \mathrm{~s}$ time out and a correction trial. Correction trials are identical repetitions of the initial presentation of a choice (Selection Trial) in which the animal responded incorrectly. A correction trial will repeat until the animal makes a correct response, and they are not used in the calculation of \% Accuracy, which only considers the accuracy of responses to selection trials. The criterion for Punish Incorrect was 100 trials in $1 \mathrm{~h}$, with accuracy greater than $80 \%$ across all selection trials (i.e. making a correct choice on greater than $80 \%$ of all selection trials).

\section{PAL training}

PAL required the animal to differentiate between two different images presented simultaneously in 2 of the 3 response windows pseudorandomly. Each image is correct only when paired with its respective location. Negative images of a flower, airplane, and spider were used as stimuli. The flower is always correct in the left position, the airplane in the centre position, and the spider in the right position. Correct responses are rewarded and punished in the same manner in the Punish Incorrect phase. Animals were trained to a criterion of 90 correct selection trials, completed within $1 \mathrm{~h}$, with greater than $80 \%$ accuracy, for two consecutive days. Correction trials were not included in the number of selection trials completed or the calculation of accuracy scores.

A schematic of the experimental timeline used in the PAL experiment is shown in Fig. 4B. After animals reached criterion in PAL, cage-mates were randomly assigned to a running wheel cage or maintained in their standard home cage. Each pair of cage-mates assigned to the running wheel condition were yoked to another pair that would be assigned to the sedentary condition in order to maintain counterbalancing of the number of pre-training days between experimental conditions. At the end of the neurogenesis protocol, all animals were food restricted to $90 \%$ of free feeding and placed in new standard home cages. Animals were given 1 day to acclimatize to the transfer procedure.

\section{PAL testing and reversal learning}

Following the neurogenesis protocol, rats' PAL retention was tested with 4 sessions of PAL. After PAL animals were moved to reversal regardless of performance and tested daily, seven days a week, for 10 days. Reversal was identical to PAL except that the correct stimuli/location pairings were changed so that the airplane was correct in the left position, the spider in the centre position, and the flower in the right position.

\section{Perfusions and immunohistochemistry}

After the conclusion of behavioral testing, rats were deeply anaesthetized and perfused intracardially. Brains were stored for $24 \mathrm{~h}$ in $4 \%$ formaldehyde at $4^{\circ} \mathrm{C}$ before being transferred to a cryoprotectant solution composed of $30 \%$ sucrose $/ 0.1 \%$ sodium azide. Tissue was sectioned using a cryostat (Leica) and 12 series of $40 \mu \mathrm{m}$ thick tissue sections were collected into an antifreeze solution composed of PBS buffered glycerol and ethylene glycol for storage at $-20{ }^{\circ} \mathrm{C}$. Immunohistochemistry was conducted to label DCX-positive neurons in the DGs. Tissue was washed 3 times in $0.1 \mathrm{M}$ PBS before being transferred to a primary antibody solution containing $0.1 \mathrm{M}$ PBS with 3\% Trition-X, 3\% Donkey Serum, and a 1:200 dilution of rabbit anti-DCX primary antibody (Product \#4604S, Cell Signalling Technology, Danvers, MA). Tissue was incubated in the primary antibody solution at room temperature for $48 \mathrm{~h}$ before being washed 3 times in $0.1 \mathrm{M} \mathrm{PBS}$ and transferred to a secondary antibody solution containing 0.1 M PBS and a 1:500 dilution of 


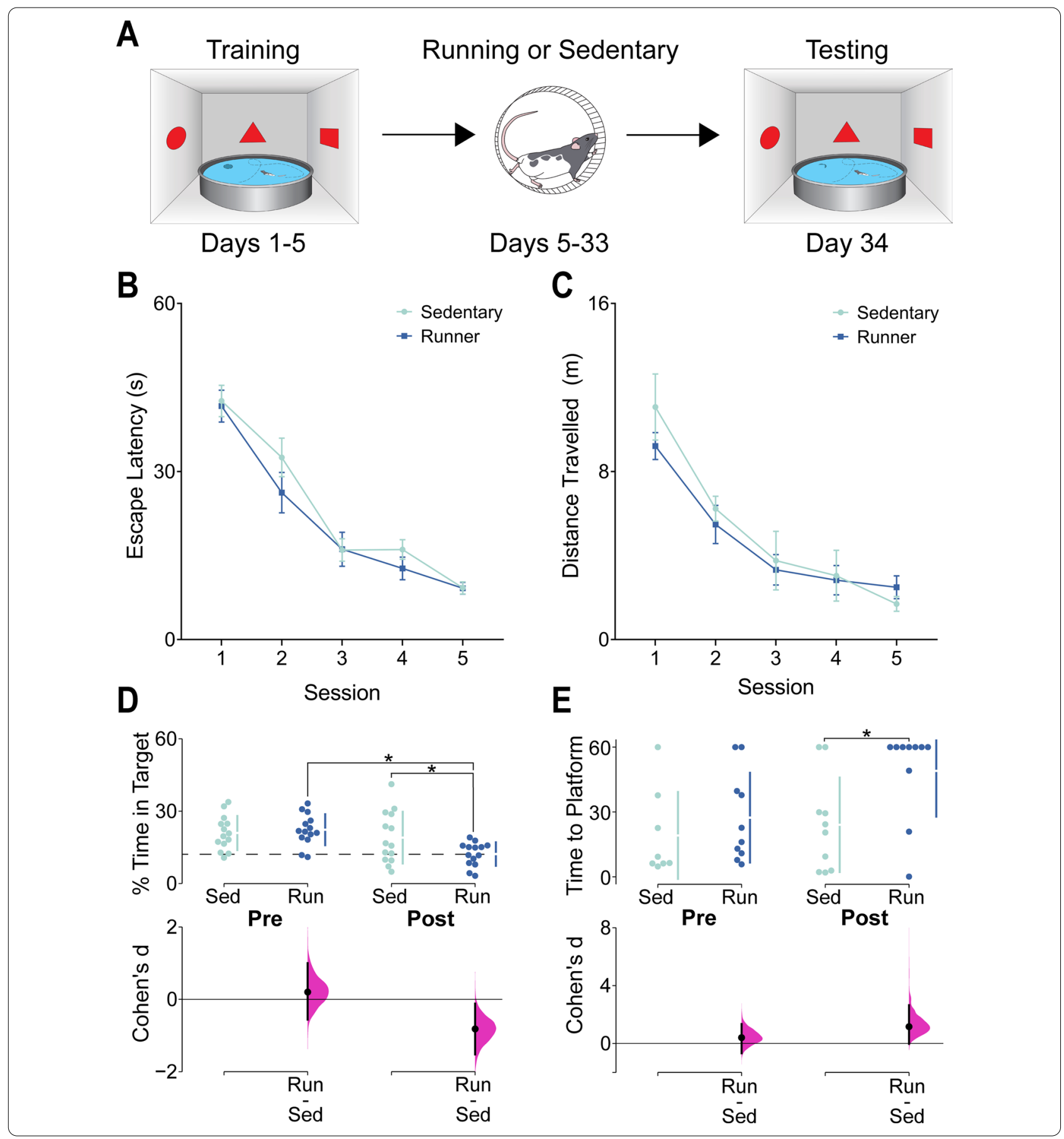

donkey anti-rabbit antibody (Dylight 488). Sections were then transferred to a solution containing $0.1 \mathrm{M}$ PBS and a 1:2000 dilution of DAPI before being mounted to glass slides and coverslipped with polyvinyl alcohol/DABCO mounting medium.

\section{Quantification of DCX}

Quantification of DCX + granule cells was performed throughout the entire rostral-caudal extent of the DG using a fluorescence microscope (Olympus FV3000) with $60 \times$ NA 1.35 oil objective. Cells were counted in the granule cell layer and the subgranular zone (defined as the $50 \mu \mathrm{m}$ zone adjacent to the granule cell layer). The area of the DG in every section was traced in order 
A

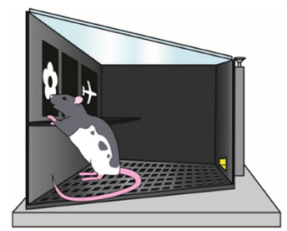

C

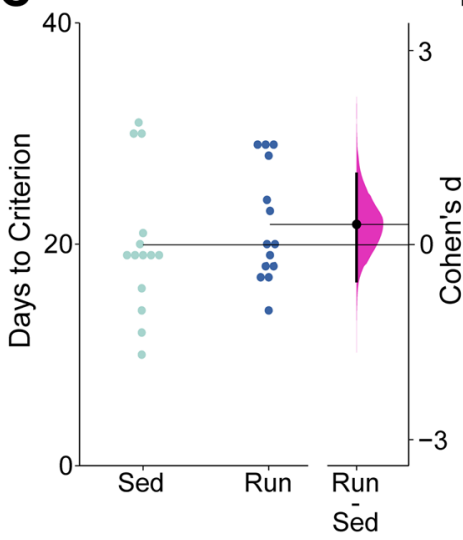

F

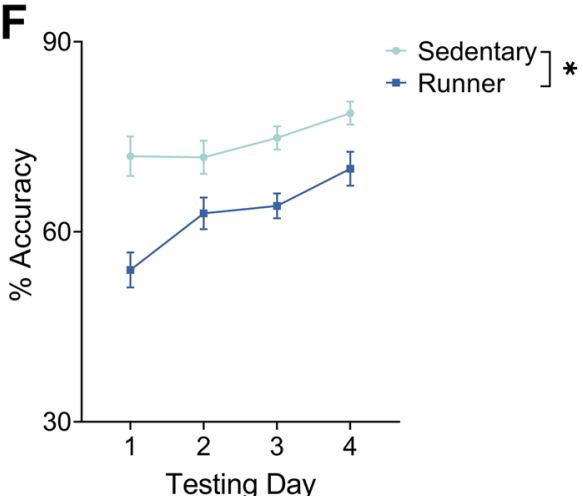

B

D
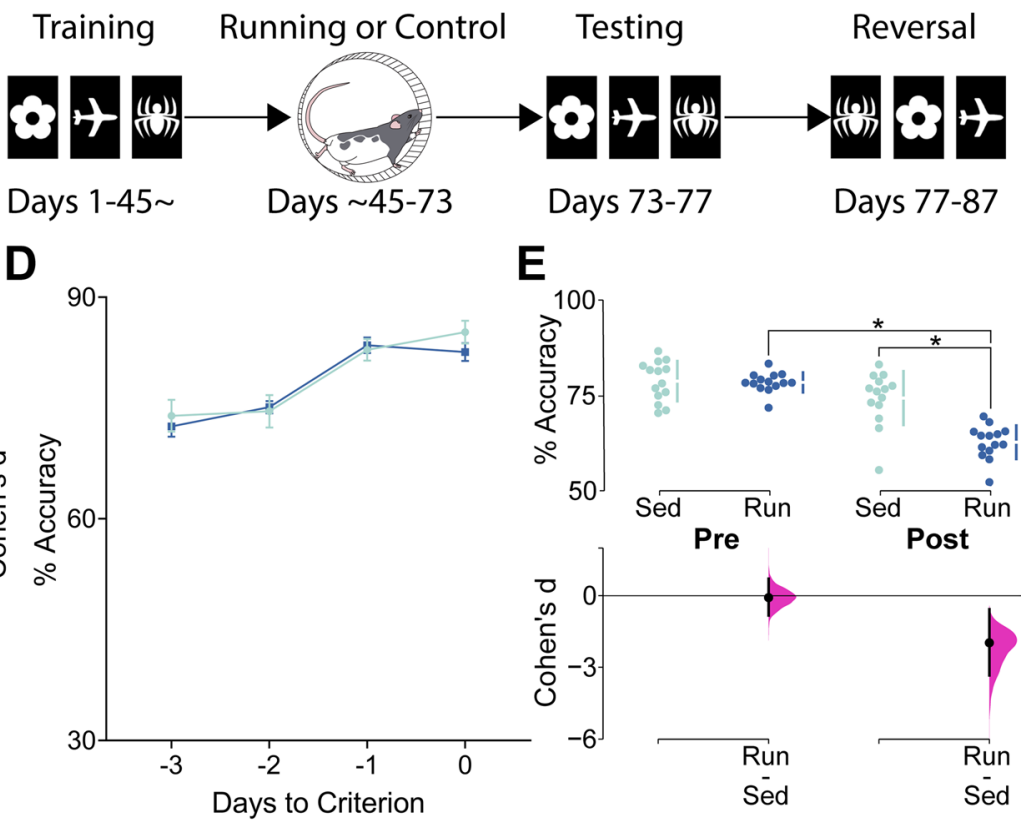

E
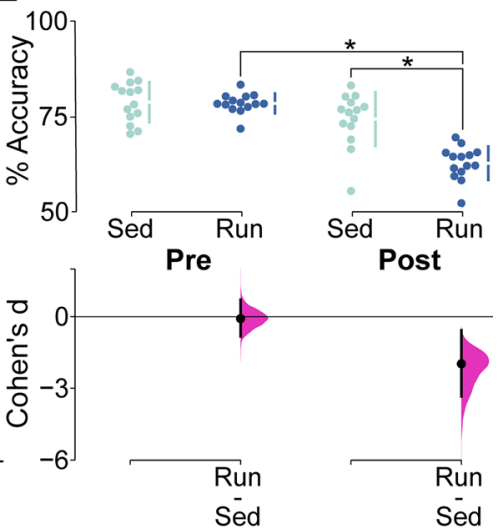

G

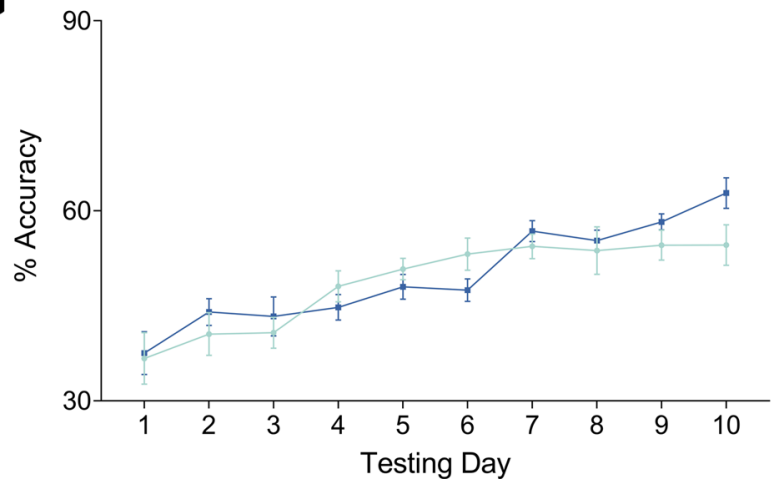

Fig. 4 A lllustration of the touchscreen apparatus used for paired associates learning (PAL) training and testing. B Timeline depicting the sequence of experiments in PAL. C Number of days to criterion for runners (Run) and sedentary controls (Sed). Data are shown as individual data points with mean (horizontal lines) and \# Days to Criterion scale on the left Y-axis. Effect size (Cohen's d) is shown as a black circle with vertical lines and gaussian distribution for the bootstrap $95 \% \mathrm{Cl}$ and Cohen's d scale on the right y-axis. Both groups reached the initial criterion in PAL at the same rate before elevation of neurogenesis. D Mean ( \pm SEM) \% Accuracy during the final 4 days of PAL training before elevation of neurogenesis. Both groups performed with the same accuracy before elevation of neurogenesis. E \% Accuracy in PAL training before (Pre) and after (Post) elevation of neurogenesis (\% Accuracy is computed as an average across 4 days of testing for each swarm plot). Upper graph shows individual data points in swarm plots with means ( \pm SD; gapped vertical line). Lower graph shows effect size (Cohen's d) as a black circle with vertical lines and gaussian distributions for the bootstrap 95\% Cl. Both groups performed with the same \% Accuracy before elevation of neurogenesis, but runners performed with significantly reduced accuracy after elevation of neurogenesis, indicating that they had forgotten the correct image-location pairings. F Mean ( \pm SEM) percent accuracy in PAL following either running or sedentary control. Runners performed with significantly and persistently reduced accuracy relative to sedentary controls over 4 days of testing. G Mean ( $($ SEM) \% Accuracy in PAL during reversal learning. Both groups performed with the same accuracy during reversal learning, indicating that running did not enhance accuracy during PAL reversal learning 
to estimate DG volume. DCX + cell counts were normalized to DG volume to control for differences in DG volume between subjects. Quantification was performed blind to treatment conditions.

\section{Experimental design and statistical analysis}

We used a combination of traditional hypothesis testing and estimation statistics, which relies on quantitative judgements of effect sizes rather than significance thresholds [20]. Continuous data such as learning curves were typically analyzed and expressed as traditional plots with standard parametric statistics, whereas post-running group differences were evaluated using combined estimation statistics and hypothesis testing. In such cases, data were represented as estimation plots with significant $\mathrm{p}$-values obtained from standard hypothesis testing and significant differences displayed on the plots. Data displayed includes individual subjects as swarm plots in addition to showing group means and standard deviation (gapped vertical line). Effect sizes are shown in separate plots as bootstrap 95\% confidence intervals and were calculated as Cohen's d. Estimation plots and statistics were generated using the Estimation Statistics website [21]. Standard hypothesis testing analyses were performed in Prism 9.

\section{Results \\ Voluntary running causes forgetting of contextual fear conditioning in a neurogenesis-dependent manner}

As expected based on previous literature, we observed significant main effects of running (Fig. 1C. $\mathrm{F}(1,52) \leq 27.79, \mathrm{p}<0.0001)$ ) and of TMZ (Fig. 1C. $\mathrm{F}(1,52) \leq 10.45, \mathrm{p}<0.0021)$ in the number of DCX + cells in the DG (Representative photomicrographs shown in Fig. 1B). We also found a significant running $\times$ treatment interaction effect $(\mathrm{F}(1,52)=4.06, \quad \mathrm{p}=0.049)$. Tukey's post hoc test demonstrated that there was a significant increase in DCX + cells in vehicle-treated runners compared to vehicle-treated sedentary mice $(p<0.0001)$. However, there was no significant increase in DCX + cells in the TMZ treated runners compared to the TMZ treated sedentary group $(p=0.11)$. Importantly, treatment with TMZ did not cause a statistically significant reduction in the amount of running (RUN $+\mathrm{VEH}$ $5.43( \pm 0.85) \mathrm{km} /$ day/rat, RUN + TMZ 3.44 $( \pm 0.52) \mathrm{km} /$ day/rat; $\mathrm{t}(10)=1.98, \mathrm{p}=0.076)$.

Prior to manipulation of neurogenesis with running/ TMZ, all 4 groups exhibited equivalent levels of freezing during CFC acquisition and there were no significant main or interaction effects (Fig. 1D: Fs $(1,52) \leq 0.53$, $\mathrm{ps} \geq 0.45)$. After manipulation of neurogenesis, we found a significant running $\times$ treatment interaction (Fig. 1E. Significant interaction of Running $\times$ TMZ: $F(1,50)=4.14$, $p=0.047)$. Post hoc tests indicated that the RUN + VEH group froze significantly less than the SED + VEH group $(p=0.0039)$, whereas this effect of running on fear memory was absent in the TMZ treated mice $(p=0.89)$.

\section{Increasing neurogenesis causes forgetting of contextual fear condition regardless of strength of training}

Having established that the forgetting effect in CFC is neurogenesis-dependent, we sought to determine whether this effect could also be modulated by the strength of CFC training. Runners and sedentary controls exhibited similar levels of freezing during conditioning in the weak training (Fig. $2 \mathrm{C}: \mathrm{t}(18)=0.53, p=0.60$ ) and strong training (Fig. 2D: $\mathrm{F}(1,54)=0.25, p=0.62$ ) conditions, although both groups in the strong training condition exhibited increased levels of freezing over successive conditioning sessions (Fig. 2D. Main effect of Day: $\mathrm{F}(2,54)=18.81, p<0.0001)$. After voluntary running (running effects on DCX shown in Additional file 1: Figure S1), runners froze significantly less than sedentary controls in both the weak and strong training conditions (Fig. 2E. Significant main effect of group: $F(1,36)=16.21$. $p=0.0003)$, indicating weakened memory for the context-fear association in runners regardless of strength of training. There was no significant interaction effect when comparing freezing in the weak and strong training conditions $(\mathrm{F}(1,36)=0.34, p=0.56)$, but a raw comparison of the effect sizes reveals that the forgetting effect in the strong training condition was of $\sim 30 \%$ lower magnitude than in the weak training condition.

\section{Increasing neurogenesis causes forgetting in the MWT}

We next examined whether running induced neurogenesis altered memory retention using a spatial version of the MWT. Prior to increasing neurogenesis, runners and sedentary controls both acquired the MWT equally with equivalent decreases in escape latency (Fig. 3B. Significant main effect of Training Day: $F(4,26)=75.16$, $\mathrm{p}<0.0001$ ) and distance travelled to the platform (Fig. 3C. Significant main effect of Training Day: $F(4,16)=25.17$, $\mathrm{p}<0.0001)$. Probe trials conducted before and after elevation of neurogenesis showed that runners exhibited a reduction in memory following 4 weeks of running but sedentary rats showed no significant change (Fig. 3D. Significant interaction of Group $\times$ Probe Trial: $\mathrm{F}(1,52)=4.07, p=0.049)$. One-sample t-tests showed that the percentage of time in the platform zone was significantly above chance level (12\%) for both groups prior to elevation of neurogenesis (Sed: $\mathrm{t}(13)=4.76, p=0.0004$; Run: $\mathrm{t}(13)=5.99, p<0.0001)$. After elevation of neurogenesis, runners spent significantly less time in the target 
zone than they did during the pre-running probe trial $(p=0.005)$ and less than sedentary controls in the postrunning probe trial $(p=0.042)$. Additionally, runners had a significantly longer latency to their first platform zone crossing in the post-running probe trial, showing that the memory impairment in runners was not due to any enhanced adaptation in platform search strategy (Fig. 3E. Significant main effect of Group: $\mathrm{F}(1,18)=5.22, p=0.03$. Significant post-neurogenesis post hoc test; $p=0.03$ ). Note that some animals $(\mathrm{n}=4$ per group) were removed from this analysis due to a tracking error in which recordings did not reliably start as soon as the rats were placed in the pool, preventing the definitive measurement of time to the first platform crossing.

\section{Increasing neurogenesis causes forgetting of PAL}

As a final test of whether neurogenesis causes forgetting, we examined the effects of increased neurogenesis on performance in PAL. Prior to elevation of neurogenesis, both groups achieved criterion in the same amount of time (Fig. 4C) and performed with nearly identical \% Accuracy in PAL (Fig. 4D, E). Analyses of pretraining Trial Completion and Response Latency are shown in Additional file 1 (Figures S2A-B. S3A-C, Table S1). After 4 weeks of voluntary exercise or sedentary control, runners exhibited a large and significant reduction in \% Accuracy (Fig. 4E. Significant interaction of Neurogenesis $\times$ Test: $\mathrm{F}(1,26)=27.10, p<0.0001)$. Moreover, the performance deficit, relative to sedentary controls, was sustained over 4 days of testing (Fig. 4D, F; Significant main effect of Neurogenesis: $\mathrm{F}(1,26)=27.29, p<0.0001$ ), although both groups also significantly improved their performance over the 4 days of retention testing, indicating significant relearning of the task (Fig. 4F; Significant main effect of Testing Day: $\mathrm{F}(3,78)=9.49, p=0.0002)$. The lack of a significant interaction of Group $\times$ Testing Day suggests that both runners and controls underwent significant improvements to \% Accuracy $(\mathrm{F}(3,78)=2.00$, $p=0.12$ ). All other analyses of post-neurogenesis PAL performance are included in Additional file 1 (Figures S2C, D, S3D, F, Table S2). Briefly, there were no differences in Trial Completion or Response Latency prior to increasing neurogenesis, but after increasing neurogenesis, runners performed more correction trials (Additional file 1: Figure S2D. Significant main effect of Group: $\mathrm{F}(1,26)=27.16, p<0.0001)$ and performed with reduced Incorrect Choice Latency (Additional file 1: Figure S4E. Significant main effect of Group: $\mathrm{F}(1,26)=6.43, p=0.02$ ). The altered Incorrect Choice Latency was not correlated with \% Accuracy $(\mathrm{r}(26)=0.04, p=0.68)$ meaning that the reduction in \% Accuracy was likely not the result of a change in response time and represents forgetting rather than a speed-accuracy trade-off effect.
Increased neurogenesis does not improve reversal learning accuracy, but increases behavioral flexibility and response speed

We also sought to determine whether increased neurogenesis would facilitate reversal learning in PAL. There were no significant differences between runners and sedentary controls in \% Accuracy (Fig. 4G; $F(1,26)=0.45$, $p=0.50)$, and no interaction of Group $\times$ Testing Day $(\mathrm{F}(9,234)=1.40, \quad p=0.18)$, indicating that increased neurogenesis did not enhance accuracy during reversal learning. However, there was a main effect of Training Day $(\mathrm{F}(9,234)=17.65, p<0.0001)$ showing that both groups significantly improved their accuracy over successive testing days. Analyses of PAL reversal learning Trial Completion and Response Latency are shown in Additional file 1 (Figures S2E, F, S3G-I, Table S3). Runners also performed with increased Selection Trials relative to sedentary controls (Additional file 1: Figure S2E. Significant main effect of Neurogenesis: $F(1,26)=26.97$, $p<0.0001)$ and reduced the number of Correction Trials they performed over successive days of reversal learning whereas sedentary controls did not (Additional file 1: Figure S3F. Significant Interaction of Neurogenesis $\times$ Testing Day: $F(9,234)=2.00, p=0.04)$. Finally, runners performed with decreased Correct Choice Latency (Figure S3G. Significant main effect of Neurogenesis: $\mathrm{F}(1,26)=24.02, \quad p<0.0001)$, Incorrect Choice Latency (Additional file 1: Figure S3H. Significant main effect of Neurogenesis: $F(1,26)=18.56, p<0.0001)$, and Reward Collection Latency (Additional file 1: Figure S3I. Significant main effect of Neurogenesis: $F(1,26)=5.50$, $p=0.027$ ). All other analyses of PAL reversal learning performance are shown in Additional file 1: Table S3.

\section{Discussion}

In the present study, we investigated the effects of increasing hippocampal neurogenesis, via voluntary running, on memory retention in three different HPCdependent memory tasks. We show here consistent evidence that voluntary running results in forgetting (the inability to recall information that was recalled previously [5]) of previously acquired memories in rats in a neurogenesis-dependent manner and that the effect extends to 3 different HPC-dependent long-term memory tasks. Several previous reports using mice, guinea pigs, and degus have demonstrated that increasing neurogenesis causes forgetting of previously acquired hippocampal memories [7-11]. However, a recent report found a null result in rats, calling into question whether the neurogenesis-induced forgetting phenomenon is present in rats and, thus, whether it is an evolutionarily conserved mechanism [12]. Our current findings show that neurogenesis-induced forgetting is clearly present in rats. 
The debate around this issue is of central importance to fields involving long-term memory research under both normal and pathological conditions. Forgetting is an essential complementary process to memory [22], aiding in the balance between plasticity and the stability of memory circuits. Thus, understanding the mechanisms underlying forgetting, including neurogenesis, is critical to the broader understanding of long-term memory function.

Neurogenesis was substantially increased by voluntary exercise, replicating a large body of previous findings [4, $7,8,12,18,19,23]$. Additionally, the running-induced increase in neurogenesis was blocked by TMZ administration in a similar fashion to previous findings $[7,8]$. Although both running and TMZ administration have effects outside of changes in neurogenesis, transgenic approaches to blocking neurogenesis have also yielded the same results [7]. Of note, TMZ in our experiment only caused a small reduction in neurogenesis in the sedentary condition that was not statistically significant. However, TMZ did significantly attenuate running-induced increases in neurogenesis, which was the critical effect for showing that forgetting was neurogenesis-dependent, rather than a consequence of some other effect of running.

We found that contextual fear memory was significantly impaired by voluntary exercise and that the effect could be blocked by treatment with TMZ to inhibit neurogenesis, replicating previous findings $[7-9,11]$. We found that both strong and weak training of the contextual fear memories resulted in neurogenesis-induced forgetting. Although the forgetting effect was of slightly lower magnitude following a strong training protocol, suggesting that more strongly trained memories may be somewhat more resilient to the effects of neurogenesis, both training protocols proved to be highly susceptible to the forgetting effect. This suggests that even highly salient memories are vulnerable to disruption by elevated neurogenesis.

In direct contrast to the results of Kodali et al. [12], we found that voluntary exercise caused forgetting of a previous platform location in the MWT, replicating previous findings that elevated neurogenesis causes retrograde impairments in spatial memory $[7,8,11]$. The rats in the Kodali et al. [12] study were administered these sessions over a period of 8 days rather than 5 days. Previous research involving HPC lesions has shown that memory acquired in more distributed learning sessions can become independent of the HPC [15]. Thus, the discrepancy here may relate to how MWT training sessions are distributed over time rather than the raw duration of training. Additionally, we measured the latency to the first platform zone crossing and found that runners were significantly impaired on this variable as well, indicating that our results did not come as a result of altered search strategy in the MWT.

A potential concern in our MWT experiment is that the use of a probe trial immediately after the conclusion of training may have induced extinction, which could be further facilitated by increased neurogenesis, leading to a nonlinear change in memory retention between groups arising from the same initial extinction learning. However, the forgetting effect is still present in the other behavioural tasks we present which contain no equivalent to a pre-running probe trial. Therefore, we deem it unlikely that the neurogenesis-mediated enhancement of extinction learning could serve as an adequate alternative interpretation of our results.

Increasing neurogenesis also caused a large decrease in accuracy of the previously acquired PAL task. It has been shown that increasing neurogenesis disrupts odor-based PAL in mice [8], but the present results are the first published account of neurogenesis-induced forgetting in the touchscreen-based PAL task. Importantly, the touchscreen-based PAL task differs from both the MWT and contextual fear conditioning in that it requires extensive training over a period of weeks but, despite this, we again show the presence of neurogenesis-induced forgetting.

Increased neurogenesis did not enhance accuracy during reversal learning, counter to previous findings that enhancing neurogenesis alleviates proactive interference [8]. This may have been related to the number of days of retention testing. Over the course of retention testing, animals in both groups showed improvements in performance consistent with relearning of the original imagelocation associations. Thus, reversal learning may have been impaired by interference from relearning during retention testing. However, runners performed significantly more trials than sedentary controls during reversal. Additionally, runners exhibited a greater reduction in the number of correction trials than controls over successive days of reversal learning. Runners also performed with lower latency than controls across all latency measures. Correction trials have previously been used as a measure of cognitive flexibility [24, 25]. The combined increase in selection trials, enhanced reduction in correction trials, and lower latencies can also be considered an indication of a general increase in task efficiency [26]. In brief, runners performed PAL reversal learning faster and more flexibly than sedentary controls, if not more accurately.

Combined, these results show that neurogenesisinduced forgetting is a clear and large effect in rats across tasks. It is worth emphasizing, however, that these results are not in contradiction with previous 
studies showing the necessity of adult-born neurons for recall of previously-encoded long-term memories. If adult-born neurons are ablated after memory formation, recall of those memories is impaired presumably because the ablated new neurons had become integrated as necessary units in the memory trace [27]. Our present claim relates specifically to the retrograde effects on long-term memories of adult-born neurons that have been newly generated after the formation of a memory trace.

In conclusion, the present study sought to extensively test whether neurogenesis-induced forgetting is present in rats. Using 3 different behavioral tasks involving different types of HPC-dependent memory with differential complexity, strength of training, and sensory modality, we show that neurogenesis-induced forgetting is robustly present in rats across HPC-dependent memory, replicating a body of previous literature in other rodent species.

\section{Abbreviations}

CFC: Contextual fear conditioning; DCX: Doublecortin; DG: Dentate gyrus; HPC: Hippocampus; MWT: Morris water task; PAL: Paired associates learning; TMZ: Temozolomide.

\section{Supplementary Information}

The online version contains supplementary material available at https://doi. org/10.1186/s13041-021-00808-4.

Additional file 1. Additional tables and figures.

\section{Acknowledgements}

The authors would like to thank Dr. Brittney R. Lins for providing the illustrations of rats and behavioural apparatus used in the figures.

\section{Authors' contributions}

JRE, JGH, GAS, and AJR designed research; GAS, DJT, AJR, KAG, APV, and JRE performed research; GAS and JRE wrote the manuscript. All authors read and approved the final manuscript.

\section{Funding}

Funding for this study was provided by an NSERC Discovery Grant (RGPIN2018-05135) to JRE and an NSERC Discovery Grant and CIHR operating Grant to JGH. GAS received funding from NSERC, the Hotchkiss Brain Institute and the Cumming School of Medicine. AJR received funding from NSERC.

\section{Availability of data and materials}

The datasets analyzed in the current study are available from the corresponding author on reasonable request.

\section{Declarations}

\section{Ethics approval and consent to participate}

All experiments were approved by the institutional animal care committees of the University of Calgary and the University of Saskatchewan and adhered to the guidelines of the Canadian Council on Animal Care.

\section{Consent for publication}

Not applicable.

\section{Competing interests}

The authors declare no competing interests.

\section{Author details}

${ }^{1}$ Department of Cell Biology and Anatomy, Hotchkiss Brain Institute, Cumming School of Medicine, HMRB 162, Health Sciences Centre, University of Calgary, 3330 Hospital Drive NW, Calgary, AB T2N 4N1, Canada. ${ }^{2}$ Yukon University, 500 University Drive, Whitehorse, YTY1A 5K4, Canada. ${ }^{3}$ Department of Anatomy, Physiology, and Pharmacology, University of Saskatchewan, 107 Wiggins Road, Saskatoon, SK S7N 5E5, Canada.

Received: 8 April 2021 Accepted: 8 June 2021

Published online: 26 June 2021

\section{References}

1. Snyder JS, Hong NS, McDonald RJ, Wojtowicz JM. A role for adult neurogenesis in spatial long-term memory. Neuroscience. 2005;130:843-52.

2. Winocur G, Wojtowicz JM, Sekeres MJ, Snyder JS, Wang S. Inhibition of neurogenesis interferes with hippocampus-dependent memory function. Hippocampus. 2006;16:296-304.

3. Arruda-Carvalho M, Restivo L, Guskjolen A, Epp JR, Elgersma Y, Josselyn SA, et al. Conditional deletion of a-CaMKII impairs integration of adult-generated granule cells into dentate gyrus circuits and hippocampus-dependent learning. J Neurosci Society for Neuroscience. 2014;34:11919-28.

4. Van Praag H, Christie BR, Sejnowski TJ, Gage FH. Running enhances neurogenesis, learning, and long-term potentiation in mice. Proc Natl Acad Sci U S A. 1999;96:13427-31.

5. Tulving E. Cue-dependent forgetting: when we forget something we once knew, it does not necessarily mean that the memory trace has been lost; it may only be inaccessible. Am Sci. 1974;62:74-82.

6. Weisz VI, Argibay PF. Neurogenesis interferes with the retrieval of remote memories: Forgetting in neurocomputational terms. Cognition. 2012;125:13-25.

7. Akers KG, Martinez-canabal A, Restivo L, Yiu AP, De Cristofaro A, Hsiang $H$ - $L$, et al. Hippocampal neurogenesis regulates forgetting during adulthood and infancy. Science. 2014;598:598-603.

8. Epp JR, Silva Mera R, Kohler S, Josselyn SA, Frankland PW. Neurogenesismediated forgetting minimizes proactive interference. Nat Commun. 2016;7:5-12.

9. Ishikawa R, Fukushima H, Frankland PW, Kida S. Hippocampal neurogenesis enhancers promote forgetting of remote fear memory after hippocampal reactivation by retrieval. Elife. 2016;5:1-17.

10. Gao A, Xia F, Guskjolen AJ, Ramsaran Al, Santoro A, Josselyn SA, et al. Elevation of hippocampal neurogenesis induces a temporally graded pattern of forgetting of contextual fear memories. J Neurosci. 2018;38:3190-8.

11. Cuartero MI, de la Parra J, Pérez-Ruiz A, Bravo-Ferrer I, Durán-Laforet V, García-Culebras A, et al. Abolition of aberrant neurogenesis ameliorates cognitive impairment after stroke in mice. J Clin Invest. 2019;129:1536-50.

12. Kodali M, Megahed T, Mishra V, Shuai B, Hattiangady B, Shetty AK. Voluntary running exercise-mediated enhanced neurogenesis does not obliterate retrograde spatial memory. J Neurosci. 2016;36:8112-22.

13. Ramírez-Amaya V, Escobar ML, Chao V, Bermúdez-Rattoni F. Synaptogenesis of mossy fibers induced by spatial water maze overtraining. Hippocampus. 1999;9:631-6.

14. Gómez-Padilla E, Bello-Medina P. Morris water maze overtraining increases the density of thorny excrescences in the basal dendrites of CA3 pyramidal neurons. Behav Brain Res. 2019;379:112373.

15. Lehmann H, Sparks FT, Spanswick SC, Hadikin C, McDonald RJ, Sutherland RJ. Making context memories independent of the hippocampus. Learn Mem. 2009;16:417-20.

16. Sutherland RJ, Sparks FT, Lehmann H. Hippocampus and retrograde amnesia in the rat model: a modest proposal for the situation of systems consolidation. Neuropsychologia Elsevier Ltd. 2010;48:2357-69.

17. Lehmann H, McNamara KC. Repeatedly reactivated memories become more resistant to hippocampal damage. Learn Mem. 2011;18:132-5. 
18. Wojtowicz JM, Askew ML, Winocur G. The effects of running and of inhibiting adult neurogenesis on learning and memory in rats. Eur J Neurosci. 2008:27:1494-502.

19. Speisman RB, Kumar A, Rani A, Foster TC, Ormerod BK. Daily exercise improves memory, stimulates hippocampal neurogenesis and modulates immune and neuroimmune cytokines in aging rats. Brain Behav Immun. 2012;28:25-43.

20. Ho J, Tumkaya T, Aryal S, Choi H, Claridge-Chang A. Moving beyond $P$ values: data analysis with estimation graphics. Nat Methods. 2019:16:565-6.

21. Estimation Statistics beta. www.estimationstats.com. Accessed Jan 2021.

22. Medina JH. Neural, cellular and molecular mechanisms of active forgetting. Front Syst Neurosci. 2018;12:1-10

23. Van Praag H, Kempermann G, Gage FH. Running increases cell proliferation and neurogenesis in the adult mouse dentate gyrus. Nat Neurosci. 1999;2:266-70.

24. Lins BR, Phillips AG, Howland JG. Effects of D- and L-govadine on the disruption of touchscreen object-location paired associates learning in rats by acute MK-801 treatment. Psychopharmacology. 2015;232:4371-82.
25. Lins BR, Howland JG. Effects of the metabotropic glutamate receptor 5 positive allosteric modulator CDPPB on rats tested with the paired associates learning task in touchscreen-equipped operant conditioning chambers. Behav Brain Res. 2016;301:152-60.

26. Roebuck AJ, Liu MC, Lins BR, Scott GA, Howland JG. Acute stress, but not corticosterone, facilitates acquisition of paired associates learning in rats using touchscreen-equipped operant conditioning chambers. Behav Brain Res Elsevier. 2018;348:139-49.

27. Arruda-Carvalho M, Sakaguchi M, Akers KG, Josselyn SA, Frankland PW. Posttraining ablation of adult-generated neurons degrades previously acquired memories. J Neurosci. 2011;31:15113-27.

\section{Publisher's Note}

Springer Nature remains neutral with regard to jurisdictional claims in published maps and institutional affiliations.
Ready to submit your research? Choose BMC and benefit from:

- fast, convenient online submission

- thorough peer review by experienced researchers in your field

- rapid publication on acceptance

- support for research data, including large and complex data types

- gold Open Access which fosters wider collaboration and increased citations

- maximum visibility for your research: over $100 \mathrm{M}$ website views per year

At BMC, research is always in progress.

Learn more biomedcentral.com/submissions 